Published in final edited form as:

J Clin Densitom. 2015 ; 18(4): 461-466. doi:10.1016/j.jocd.2015.04.012.

\title{
The epidemiology of sarcopenia
}

\author{
Dr. Richard Matthew Dodds ${ }^{*}, 1,2$, Dr. Helen Clare Roberts ${ }^{1,2,3,4}$, Prof. Cyrus Cooper ${ }^{1,3,5}$, and \\ Prof. Avan Aihie Sayer $1,2,3,4,6$ \\ ${ }^{1}$ Medical Research Council Lifecourse Epidemiology Unit, University of Southampton \\ ${ }^{2}$ Academic Geriatric Medicine, Faculty of Medicine, University of Southampton \\ ${ }^{3}$ National Institute for Health Research Southampton Biomedical Research Centre, University of \\ Southampton and University Hospital Southampton NHS Foundation Trust \\ ${ }^{4}$ National Institute for Health Research Collaboration for Leadership in Applied Health Research \\ and Care: Wessex \\ ${ }^{5}$ National Institute for Health Research Musculoskeletal Biomedical Research Unit, University of \\ Oxford
}

${ }^{6}$ Newcastle University Institute of Ageing and Institute of Health and Society, Newcastle University

\section{Abstract}

The aim of this review is to describe the epidemiology of sarcopenia, specifically prevalence, health outcomes and factors across the life course that have been linked to its development.

Sarcopenia definitions involve a range of measures (muscle mass, strength and physical performance) which tend to decline with age and hence sarcopenia becomes increasingly prevalent with age. Less is known about prevalence in older people in hospital and care homes, although it is likely to be higher than in community settings. The range of measures used, as well as the cutpoints suggested for each, presents a challenge for comparing prevalence estimates between studies. The importance of sarcopenia is highlighted by the range of adverse health outcomes that strength and physical performance (and to a lesser extent, muscle mass) have been linked to. This is shown most strikingly by the finding of increased all-cause mortality rates among those with weaker grip strength and slower gait speed. A life course approach broadens the window for our understanding of the aetiology of sarcopenia and hence the potential intervention. An example is physical activity, with increased levels across mid-adulthood appearing to increase muscle mass and strength in early old age. Epidemiological studies will continue to make an important contribution to our understanding of sarcopenia and possible avenues for intervention and prevention.

*Corresponding author: Medical Research Council Lifecourse Epidemiology Unit, Southampton General Hospital, Southampton SO16 6YD, UK.rd@mrc.soton.ac.uk.

Disclosures - none of the authors have declared any conflicts of interest. 


\section{Introduction}

The term sarcopenia was initially used to describe the loss of muscle mass with age and more recent definitions have come to incorporate the loss of muscle strength and physical performance ${ }^{1}$. Its importance is highlighted by findings such as those of middle and older ages with weaker grip strength have, on average, shorter survival times than stronger individuals ${ }^{2}$. There are a wealth of epidemiological studies that have investigated risk factors for, and consequences of, low muscle mass, strength and physical performance. The aim of this article is to describe the epidemiology of sarcopenia, both in terms of individual measures and the more recently developed diagnostic criteria. We begin by considering the prevalence of sarcopenia.

\section{Prevalence}

This section aims to address the questions: how common is sarcopenia, and how does its prevalence vary with age and setting? To do this, we draw on results from studies of components of sarcopenia: muscle mass, muscle strength and physical performance, as well as studies combining these measures using the European Working Group on Sarcopenia in Older People (EWGSOP) definition.

Cut-points have been proposed for what constitutes pathologically low values for these measures. These cut-points have been derived in two ways: firstly by considering the normal range encountered at the peak of function in young adult life. This is analogous to the Tscore approach used for measurements of bone density in the diagnosis of osteoporosis in women and is illustrated in Figure 1. The second approach has been to select cut-points based on the optimum balance of sensitivity and specificity for predicting a relevant outcome, such as mobility disability. As the population average value for a given measure declines with age, the proportion of individuals below a given cut-point increases (also illustrated in Figure 1). So as one would expect, the prevalence of sarcopenia increases with age.

\section{Muscle mass}

In order to estimate the prevalence of low muscle mass, sufficiently large samples of the general population are required. Techniques for assessing muscle mass in such settings include anthropometry, bioelectrical impedance (BIA) and dual energy x-ray absorptiometry (DXA). Anthropometric measures may be prone to error in older people ${ }^{1}$. BIA produces estimates of total fat mass and non-fat mass and has the advantage over DXA that the equipment used is portable. However it has been questioned to what extent BIA provides additional information beyond that from anthropometric measurements. DXA is able to divide total body mass into estimates of fat mass, bone mass and lean mass (which includes muscle tissue and solid organs). DXA has the advantage that its estimates can be restricted to an area of the body, such as the arms and legs and hence avoid measuring the lean mass of the solid organs. This section now focuses on estimates of the prevalence of low muscle mass from DXA scans. 
Cut-points for DXA have typically come from young adult values, specifically two standard deviations (SDs) below the sex-specific young adult mean appendicular lean mass (ALM) divided by height squared. Example cut-points are $7.23 \mathrm{~kg} / \mathrm{m}^{2}$ in males and $5.67 \mathrm{~kg} / \mathrm{m}^{2}$ in females. Applying these cut-points to older populations gives estimates of prevalence such as of $20 \%$ of those aged $70-79$ and $30 \%$ of those aged over 80 .

More recently, the FNIH Sarcopenia Project have proposed cut-points for ALM from DXA based on its relationship with weak grip strength at ages 65 and older. Specifically, ratios of ALM to BMI of below 0.789 in men and 0.512 in women were found to provide the optimum balance of sensitivity and specificity for the detection of weak grip strength. The prevalence in their sample below these cut-points was $20 \%$ of men and $16 \%$ of women.

\section{Muscle strength}

Several measures exist for the measurement of muscle strength. Grip strength has been recommended as the most practical method of measuring muscle strength in the clinical setting ${ }^{1}$ and has been found to correlate physical performance measures in the lower limbs. Data from the inCHIANTI have been used to produce grip strength cut-points two standard deviations below a gender-specific young adult mean, showing a high prevalence of weak grip at age 65-74: around $60 \%$ of men and $40 \%$ of women fell below cut-points of approximately 40 and $18 \mathrm{~kg}$, respectively. In the same study, the ROC method was used to identify optimal cut-points for the detection of slow gait speed in older people of $30 \mathrm{~kg}$ in men and $19 \mathrm{~kg}$ in women, although the prevalence of those at or below these values was not stated.

The FNIH Sarcopenia Project found that cut-points of $26 \mathrm{~kg}$ in men and $16 \mathrm{~kg}$ in women best identified individuals with mobility disability (assessed using slow gait speed) at ages 65 and older $^{3}$. The prevalence below these cut-points in their community-dwelling sample was 5\% per cent of men and $18 \%$ of women. Mean grip values for hospitalised older patients admitted for rehabilitation and nursing home residents suggest that the majority of individuals in these groups fall below the cut-points described in this section ${ }^{4}$.

\section{Physical performance}

The most commonly described measure of physical performance in the assessment of sarcopenia is gait speed. Other measures include standing balance and chair rise times, which can be combined with gait speed in the form of the Short Physical Performance Battery, the results of which are predictive of ageing outcomes. However there is also evidence that gait speed alone may have similar predictive power to the complete battery of tests.

Gait speed can be assessed in the clinical setting by measuring the time taken to walk a set distance, for example $4 \mathrm{~m}$, at usual pace. Various cut-points have been proposed, with a gait speed $0.8 \mathrm{~m} / \mathrm{s}$ or greater being a useful level which has been shown to predict survival approximately at or above the median level for an individual's age and gender. Estimates of the prevalence of gait speed below $0.8 \mathrm{~m} / \mathrm{s}$ vary; a Spanish study found that $56 \%$ of men and women aged 75 and over fell below this level. 


\section{Combined measures}

The EWGSOP has defined sarcopenia as low muscle mass in combination with either poor physical performance or weak muscle strength ${ }^{1}$. In an English cohort study, the prevalence of sarcopenia using the EWGSOP definition was found to be $4.6 \%$ of men and $7.9 \%$ of women at mean age $67^{5}$. A recent review considered this and 17 other studies from mainly community-dwelling populations in Europe, the USA and Japan which had also estimated the prevalence of sarcopenia using the EWGSOP definition. Comparison of results of across these studies was difficult due to the varying measures and cut-points used for muscle mass, physical performance and muscle strength, as well as inconsistent approaches to reporting results for different age groups.

\section{Summary for this section}

This section has shown the variety of approaches used to estimate the prevalence of sarcopenia. Firstly, cut-points for individual components of sarcopenia have been produced, using both young adult values and also associations with relevant outcomes such as mobility disability. Cut-points (and corresponding prevalence estimates) from the latter approach tend to be lower than those from the former; using relevant outcomes to define cut-points may therefore help better identify individuals with accelerated loss of muscle mass and function. Secondly, the EWGSOP combined definition for sarcopenia is now being applied in prevalence studies, although differences in the implementation of the definition make comparison between these studies difficult.

A common finding from these approaches is that the prevalence of sarcopenia appears to increase with age. Few studies have examined prevalence in hospitalised older people and care home residents, although the prevalence in these settings is likely to be far higher than in community-dwelling populations. We now move on to consider findings from epidemiological studies that highlight the potential impact of sarcopenia on an older person's subsequent health.

\section{Major health outcomes associated with sarcopenia}

This section aims to address the question: why does sarcopenia matter? To do this, we review evidence from observational studies linking components of sarcopenia as well as the EWGSOP definition to subsequent outcomes, as summarised in Figure 2. By doing so, we hope to highlight the potential of the measurements described in the previous section for identifying individuals who may be at risk of adverse health in the future.

\section{Muscle mass}

Low muscle mass has previously been associated with disability. In the NHANES III study, cut-points for lean mass measured using BIA were developed to identify men and women at high-risk of disability in a cross-sectional manner. This association with disability was then used to estimate the economic impact of sarcopenia in the United States in the year 2000 as approximately $\$ 18.5$ billion. 
However a subsequent review of the results from NHANES III and eight other studies did not find evidence of a consistent relationship between low muscle mass and disability, unlike that found for low muscle strength ${ }^{6}$. Similarly, low muscle mass has not been found to be predictive of mortality. These findings explain why the term sarcopenia has been extended from solely the loss of muscle mass with age to include the age-related losses of muscle strength and physical performance, collectively referred to as function.

Even though studies to date do not show clear links between muscle mass and subsequent health, it is important to note that muscle tissue forms a reservoir essential during periods of acute illness. Our understanding of the role of muscle mass is likely to increase as techniques for its measurement are incorporated into more large-scale population studies.

\section{Muscle strength}

The ease of measuring grip strength using a handheld dynamometer has led to its inclusion in many epidemiological studies as a proxy measure of overall muscle strength. This has allowed associations between muscle strength and health to be explored. For example, two systematic reviews and subsequent studies have shown that weaker grip strength is associated with an increased risk of incident or worsening disability in activities of daily living.

Weak grip strength has also been associated with subsequent morbidity, including increased risk of subsequent fracture and cognitive decline. Cross-sectional relationships have also been found with the metabolic syndrome and impaired glucose tolerance ${ }^{7}$. Finally weak grip strength during hospital admission in older men has been associated with functional decline on discharge, as well as a reduced likelihood of discharge to their usual place of residence following a period of in-patient rehabilitation ${ }^{8}$.

Perhaps the most striking findings from epidemiological studies of health outcomes associated with weak grip strength are those on survival times. A previous meta-analysis combined results from 14 studies, including four studies with a mean age under 60 at baseline and seven with over 10 years of follow-up ${ }^{2}$. This showed that those in the lowest quarter of grip strength were at over one-and-a-half times the risk of death during follow-up compared to those in the highest quarter (summary hazard ratio 1.67 [95\% CI: 1.45, 1.93], including adjustment for age, sex and body size).

\section{Physical performance}

Gait speed has also been included in many epidemiological studies as a measure of physical performance. There is evidence of similar associations between slow gait speed and subsequent disability and morbidity as described above for weak grip strength.

Slower gait speed has also been shown to predict shorter survival times. An analysis of data from nine studies of adults aged 65 and above was used to estimate survival by gait speed for different age groups. For example, men aged 75-84 were found to have an overall median 5 -year survival of 74\%; this fell to $60 \%$ in those with gait speed below $0.4 \mathrm{~m} / \mathrm{s}$ and rose to $93 \%$ in those with gait speed above or equal to $1.4 \mathrm{~m} / \mathrm{s}^{9}$. Gait speed was also found to have similar predictive power for mortality as a range of factors including chronic diseases and 
prior hospitalisation. It has therefore been suggested that gait speed could be a useful clinical tool for identifying those at increased risk of death, for whom the risks of longer-term preventative strategies are likely to outweigh the benefits.

\section{Combined measures}

Few studies have so far tested the ability of the EWGSOP definition of sarcopenia to identify individuals at risk of adverse outcomes; one might expect it to be effective in this regard, given the associations that have already been described between the individual components of the EWGSOP definition (especially muscle strength and physical performance) and ageing outcomes. Indeed there is recent evidence from the inCHIANTI study that meeting the criteria of the EWGSOP definition is associated with increased likelihood of subsequent disability, hospitalisation and mortality.

A question relevant to the clinical setting is to whether assessing the three components of EWGSOP sarcopenia is more informative than using a single measure. There is evidence that grip strength and gait speed make independent contributions to mortality prediction. The situation for muscle mass is currently less clear, however. The findings from the prevalence section of this review also suggest that large numbers of older people may fall below strength and performance cut-offs, and therefore require measurement of muscle mass by the EWGSOP definition. There has been concern raised about whether this would be feasible.

\section{Summary for this section}

This section has summarised findings from observational epidemiological studies which demonstrate the adverse health outcomes associated with sarcopenia. Muscle strength and physical performance, which can be assessed using grip strength and gait speed, respectively, are recognised to have stronger associations with subsequent health than muscle mass. They may therefore have a role as clinical tools for predicting an individual's risk of adverse outcomes.

\section{A life course approach to understanding sarcopenia}

So far, this review has shown that sarcopenia is prevalent and associated with a range of important ageing outcomes. There is therefore considerable interest in developing effective interventions for older people with sarcopenia. The role of exercise interventions is well established and they are shown to prevent subsequent disability.

A complementary approach considers aetiological factors for sarcopenia across the entire life course, and by doing so substantially broadens the window for potential interventions to prevent or delay the development of sarcopenia. The life course epidemiology framework views function in old age as the combination of two phases: growth to the peak obtained in early adult life and subsequent decline thereafter, as shown in Figure 1. This final section of the review considers the range of factors (summarised in Figure 2) that have been linked to muscle mass and strength during these two phases. 


\section{Factors associated with growth to peak muscle mass and strength}

Genetic and early life factors-Twin studies suggest that a substantial proportion (30\% in one study) of the variance in muscle mass and strength may be explained by genetic factors. Genes related to the myostatin pathway have been linked to knee strength in young men, although an association with muscle mass, assessed using anthropometry, was not seen.

Early growth is also associated with subsequent muscle strength and mass. Data from several cohort studies have been used to investigate relationships between birth weight (as a proxy measure of intrauterine development) and grip strength. A systematic review and meta-analysis of 13 such studies, including nine with participants at mean age under 40, showed a small but consistent effect: each extra kg of birth weight was associated an average increase of $0.86 \mathrm{~kg}$ in grip strength after adjustment for age and height ${ }^{10}$. A similar positive relationship has been shown between birth weight and lean mass in children and adolescents. Finally there is evidence that faster rates of growth in the first two years of life are associated with greater muscle strength in children and young men.

Diet and physical activity-The role of diet in infancy has been examined as a potential factor contributing to peak muscle mass and strength. For example, there is evidence that longer duration of breastfeeding is associated with increased lower body explosive strength (assessed by standing long jump tests) in adolescence. In the Southampton Women's Survey, children's weaning diets were scored on their compliance with guidelines: those in the top quarter were found to have higher lean mass assessed by DXA at age 4 than those in the bottom quarter. Finally less is known about the relationship between physical activity and muscle mass and strength in childhood, although we know from cross-sectional studies that more active children tend to have lower fat mass and higher grip strength.

\section{Factors associated with the age-related decline in muscle mass and strength}

Genetic and early life factors-Genetic and early life factors have also been investigated in ageing cohorts that have measured muscle mass and strength during the decline phase. Genetic factors have included negative findings for associations between polymorphisms of the GH/IGF axis and grip strength. Birth weight has been positively associated with grip strength across several studies (as described above) and with muscle size as assessed by peripheral computed tomography scans. Longer duration of breastfeeding has also been linked to higher grip strength in men at mean age 66 in the Hertfordshire Cohort Study. Taken together, these results highlight the potential importance of early life factors for the development of sarcopenia in older people.

Diet and physical activity-Observational studies suggest that a wide range of dietary factors in older people may be important for the development of sarcopenia. Greater dietary protein intake has been associated with reduced loss of ALM over a 3 year period in an observational study, although a Cochrane review of protein supplementation found no consistent effect on functional measures relevant to sarcopenia such as grip strength and only a very small effect on arm muscle circumference. The current situation for vitamin $\mathrm{D}$ is similar, with positive associations from observational studies not consistently replicated in intervention studies. One challenge for observational studies is the tendency for intake of 
dietary components to be highly correlated with one another; an alternative approach may therefore be whole-diet interventions which attempt to change overall dietary patterns.

Exercise interventions for older people of relatively short time duration, typically $2-3$ months, show the benefits of particular types of structured physical activity for sarcopenia. Observational studies can provide additional information on the potential effects of the types of activity that people generally undertake and also examine relationships across the life course. For example, in the MRC National Survey of Health and Development, a cumulative benefit of increased leisure time physical activity across ages 36, 43, 53 and 60-64 has been found for grip strength at age 60-64 years: those in the most active third were on average $2.11 \mathrm{~kg}(95 \%$ CI: $0.88,3.35)$ stronger than those in the least third ${ }^{11}$. Similar benefits have been found for appendicular lean mass at the same age.

Chronic conditions and hormones-As described in the health outcomes section, a variety of conditions have been considered as an outcome of sarcopenia. It is likely that chronic illnesses also contribute to the development of sarcopenia. For example, raised serum creatinine has been linked to loss of lean mass in men and impaired glucose tolerance has been associated with weaker grip strength ${ }^{7}$. Medications have also been investigated, with nitrates and ACE inhibitors being linked to reduced and increased strength, respectively. Finally observational studies suggest a role for inadequate testosterone and growth hormone, although corresponding intervention trials have not suggested these can be safely used for the treatment of sarcopenia ${ }^{12}$.

\section{Summary for this section}

This section has outlined how taking a life course approach allows us to consider the role of a wide range of factors associated with the growth to peak muscle mass and strength and the subsequent age-related decline. A limitation is that the findings discussed in this section are mainly observational and may therefore have alternative explanations such as residual confounding and reverse causality. However an advantage of this approach remains that it allows relationships to be investigated over a longer time course than would be possible using the conventional trial design.

\section{Conclusions}

This review has looked at the current understanding about the prevalence and health consequences of sarcopenia as well as taking a life course approach to understanding its development. Numerous epidemiological studies have included measures relevant to sarcopenia in their assessments and hence they will continue to make a marked contribution to our understanding of how sarcopenia develops, its assessment in the clinical setting and potential new directions for management.

\section{References}

1. Cruz-Jentoft AJ, Baeyens JP, Bauer JM, et al. Sarcopenia: European consensus on definition and diagnosis: Report of the European Working Group on Sarcopenia in Older People. Age Ageing. 2010; 39(4):412-23. [PubMed: 20392703] 
2. Cooper R, Kuh D, Hardy R, Mortality Review Group. Objectively measured physical capability levels and mortality: systematic review and meta-analysis. BMJ. 2010; 341:c4467. [PubMed: 20829298]

3. Alley DE, Shardell MD, Peters KW, et al. Grip strength cutpoints for the identification of clinically relevant weakness. J Gerontol A Biol Sci Med Sci. 2014; 69(5):559-66. [PubMed: 24737558]

4. Roberts HC, Syddall HE, Sparkes J, et al. Grip strength and its determinants among older people in different healthcare settings. Age Ageing. 2013:1-6. [PubMed: 23926093]

5. Patel HP, Syddall HE, Jameson K, et al. Prevalence of sarcopenia in community-dwelling older people in the UK using the European Working Group on Sarcopenia in Older People (EWGSOP) definition: findings from the Hertfordshire Cohort Study (HCS). Age Ageing. 2013; 42(3):378-84. [PubMed: 23384705]

6. Manini TM, Clark BC. Dynapenia and aging: an update. J Gerontol A Biol Sci Med Sci. 2012; 67(1):28-40. [PubMed: 21444359]

7. Aihie Sayer A, Dennison EM, Syddall HE, Gilbody HJ, Phillips DIW, Cooper C. Type 2 diabetes, muscle strength, and impaired physical function: the tip of the iceberg? Diab Care. 2005; 28(10): 2541-2. [PubMed: 16186295]

8. Roberts HC, Syddall HE, Cooper C, Aihie Sayer A. Is grip strength associated with length of stay in hospitalised older patients admitted for rehabilitation? Findings from the Southampton grip strength study. Age Ageing. 2012; 41(5):641-6. [PubMed: 22777206]

9. Studenski S, Perera S, Patel K, et al. Gait speed and survival in older adults. JAMA. 2011; 305(1): 50-8. [PubMed: 21205966]

10. Dodds R, Denison HJ, Ntani G, et al. Birth weight and muscle strength: a systematic review and meta-analysis. J Nutr Heal Ageing. 2012; 16(7):609-15. [PubMed: 22836701]

11. Dodds R, Kuh D, Aihie Sayer A, Cooper R. Physical activity levels across adult life and grip strength in early old age: updating findings from a British birth cohort. Age Ageing. 2013; 42(6): 794-798. [PubMed: 23981980]

12. Aihie Sayer A, Robinson SM, Patel HP, Shavlakadze T, Cooper C, Grounds MD. New horizons in the pathogenesis, diagnosis and management of sarcopenia. Age Ageing. 2013; 42(2):145-50.

[PubMed: 23315797] 


\section{Key points box}

- Sarcopenia definitions involve a range of measures (muscle mass, strength and physical performance) which tend to decline with age and hence sarcopenia becomes increasingly prevalent with age. Less is known about prevalence in older people in hospital and care homes, although it is likely to be higher than in community settings.

- The importance of sarcopenia is highlighted by the range of adverse health outcomes that strength and physical performance (and to a lesser extent, muscle mass) have been linked to. This is shown most strikingly by the association of all-cause mortality with weak grip strength and slow gait speed.

- $\quad$ A life course approach broadens the window for our understanding of the aetiology of sarcopenia and hence the potential intervention. An example is physical activity, with increased levels across midadulthood appearing to increase muscle mass and strength in early old age. 


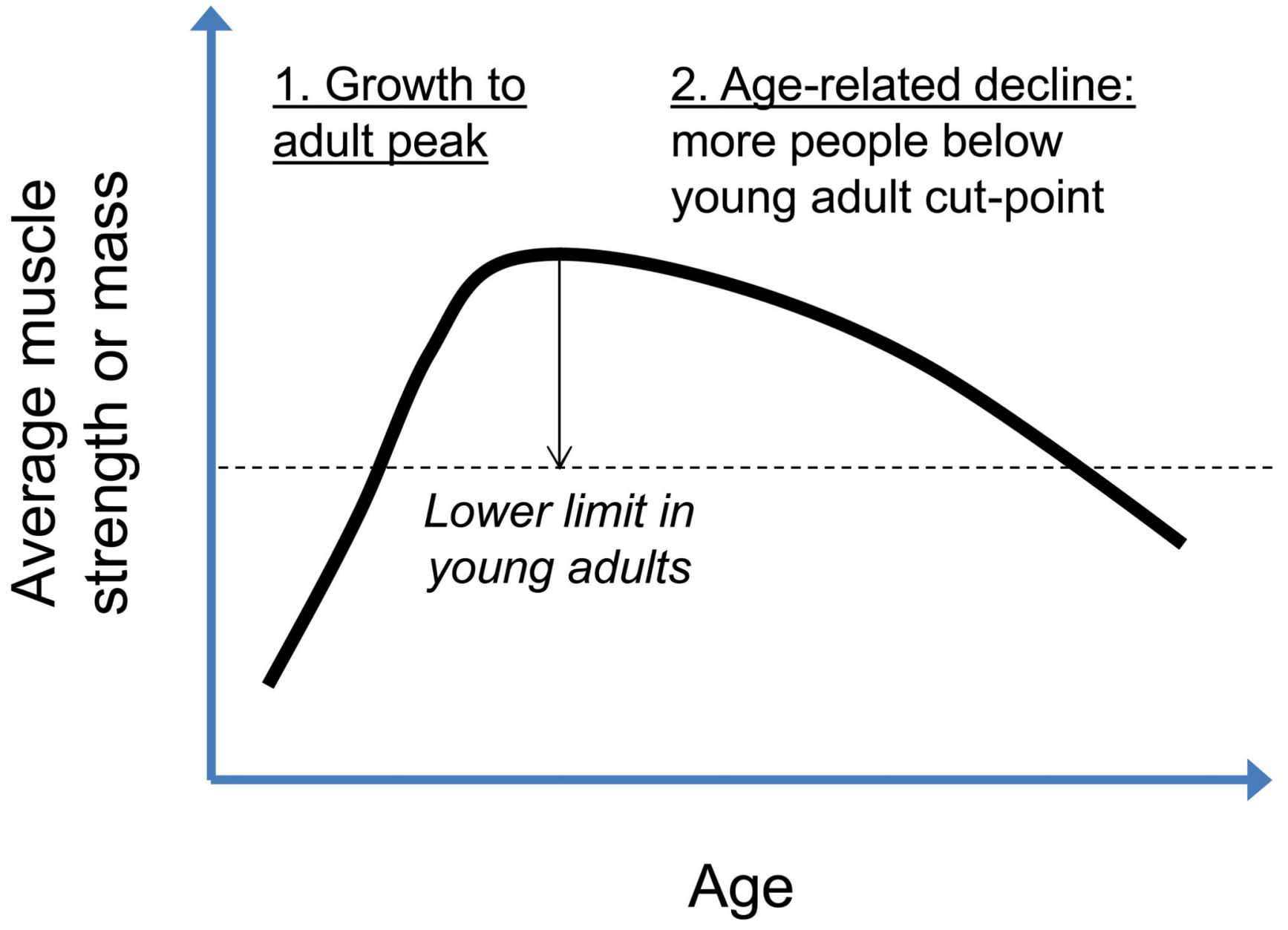

Figure 1.

A life course approach to sarcopenia

On average, peak muscle mass and strength are reached in early adult life prior to decline. The dashed line shows the use of the normal range encountered in young adult life (analogous to the T-score approach in osteoporosis) to produce cut-points for low muscle mass or strength. The prevalence below this cut-point inevitably rises with age, as average mass or strength declines. 


\begin{tabular}{|c|c|c|}
\hline $\begin{array}{l}\text { Factors across the life } \\
\text { course: } \\
\text { - } \quad \text { Genetic factors } \\
\text { - } \text { Birth weight / } \\
\text { early growth } \\
\text { - Diet } \\
\text { - Physical activity } \\
\text { - Chronic illness } \\
\text { - Hormonal changes }\end{array}$ & $\begin{array}{c}\text { Sarcopenia: } \\
\text { The loss of } \\
\text { muscle mass } \\
\text { and function } \\
\text { with age }\end{array}$ & $\begin{array}{l}\text { Health outcomes: } \\
\text { - } \quad \text { Disability } \\
\text { - } \quad \text { Morbidity including } \\
\text { fracture and cognitive } \\
\text { decline } \\
\text { - } \quad \text { Length of hospital } \\
\text { stay } \\
\text { - Shorter survival times }\end{array}$ \\
\hline
\end{tabular}

Figure 2.

Sarcopenia: major health outcomes and risk factors

Several factors across the life course have been associated with muscle mass and strength and hence are likely to contribute to the development of sarcopenia, which in turn is recognised as having a range of adverse health outcomes. 